\title{
EFECTOS DE LA ACTIVACIÓN CONDUCTUAL EN LA CALIDAD DE VIDA Y ESTADO EMOCIONAL DE LOS PACIENTES CON CÁNCER DE PULMÓN
}

\author{
EFFECTS OF THE BEHAVIORAL ACTIVATION IN THE LIFE QUALITY AND \\ EMOTIONAL STATE FOR LUNG CANCER PATIENTS
}

\section{Concepción Fernández Rodríguez', Erica Villoria Fernández², Paula Fernández García y Sonia González Fernández'}

1 Departamento de Psicología. Universidad de Oviedo

2 Universidad Autónoma de Chile

Resumen

Objetivo: Desde un análisis contextualfuncional de los problemas/limitaciones del paciente oncológico, se valora el efecto en la calidad de vida y estado emocional de una intervención centrada en la Activación Conductual con enfermos de cáncer de pulmón durante el tratamiento oncológico.

Método: 90 pacientes de reciente diagnóstico fueron asignados aleatoriamente a una condición experimental (G.E.: $N=50$ ) de 4 sesiones individuales orientadas a restablecer actividades relevantes, eliminar conductas de enfermedad y modificar el patrón de evitación experiencial; y a una condición control (G.C.: $\mathrm{N}=40$ ) que ocupó el mismo número de sesiones en la evaluación de la calidad de vida sin ofrecer asesoramiento o tratamiento psicológico. Todos los participantes completaron, en cada sesión y en seguimiento trimestral, las escalas QLQ-C30, HAD, IK, EG y SP. Se empleó un diseño de medidas parcialmente repetidas. La evolución y tendencia de las variables se realizó mediante MLG y MLM.

Resultados: Los participantes resultaron representativos de la población estudiada y no difirieron entre ellos en el pre-tratamiento. En ambos grupos se produjo una pérdida elevada de sujetos por hospitalización/fallecimiento. EI G.E. mostró, a lo largo del tiempo, mejoría estadísticamente significativa $(p<0,05)$ en todas las subescalas del QLQ-C30. En las subescalas
Abstract

Objective: Based on a functional-contextual analysis of the problems cancer patients have, the efficacy of Behavioral Activation Therapy for the prevention of emotional disorders and the promotion of life quality on lung cancer patients will be analyzed.

Method: A total of 90 lung cancer patients were selected consecutively. Patients were randomly assigned to un experimental group (G.E.: $N=50$ ) where a specific protocol was designed to increase relevant activities and healthy behaviors, erasing disease behaviors previously learned and modifying the pattern of experiential avoidance; and a control group (G.C.: $N=40$ ) where life quality and psychosocial effects of both illness and treatment were assessed, without ever offering counseling or psychological treatment. Both conditions received a total of four individual one hour long treatment sessions in the hospital. Results were collected through standardized scales (IK, HADS, QLQ-C30, EG y SP) in each of the treatment sessions and during the three months follow-ups. The evolution and tendency of the variables was analyzed using MLG y MLM.

Results: Participants, doe to their clinical and sociodemographic characteristics resulted a representative group of the population studied and did not differ between themselves in the pretreatment. In both groups there was a loss of subjects produced by hospitalization / death. The

Correspondencia:

Concepción Fernández Rodríguez

Dpto. de Psicología. Universidad de Oviedo. Plaza Feijoo, s/n - 33005 Oviedo

E-mail: frconcha@uniovi.es 
HAD, se alcanzaron diferencias significativas entre grupos a favor del G.E $(p<0,05)$. Los beneficios del G.E. se debilitaron en el seguimiento. El GC no experimentó cambios.

Conclusiones: Se aportan evidencias del interés de los objetivos y procedimientos de Activación Conductual para mejorar, durante el tratamiento oncológico, la calidad de vida y estado emocional. Se señala la necesidad de indagar las características de los pacientes y/o de la intervención de las que depende su eficacia y eficiencia.

Palabras clave: Terapia de Activación Conductual, cáncer, calidad de vida, ansiedad, depresión. comparison analysis inter and intra groups indicate that among participants, the G. E. showed, over time, statistically significant differences $(p<0.05)$ in all subscales of QLQ- C30 functioning. In the HAD subscales, significant differences between groups in favor of GE $(p<0.05)$ were achieved. Improvements shown by the G.E. weakened during the follow-up.

Conclusions: Behavioral Activation Therapy, during cancer treatment, improved life quality and emotional adjustment. The study indicates the need to investigate the characteristics of patients and/or intervention, as its effectiveness and efficiency depends on them.

Key words: Behavioral Activation Therapy, cancer, life quality, anxiety, depression.

\section{INTRODUCCIÓN}

El apoyo material y social es una condición directamente relacionada con el afrontamiento efectivo de la enfermedad oncológica y con la calidad de vida de los pacientes $^{(1,2)}$. Sin embargo, en ocasiones y con el propósito de minimizar al enfermo sus padecimientos, familiares y sanitarios le facilitan un abandono progresivo de las actividades cotidianas, más allá de lo que justificaría su estado físico. Así, en un afán por sentirse bien y tal y como se le insta socialmente, la persona puede delegar o abandonar tareas y responsabilidades cotidianas y apartarse $\mathrm{O}$, al menos intentarlo, de experiencias, preocupaciones o pensamientos negativos. Aunque de forma inmediata pueda sentirse aliviado, con el abandono/evitación de actividades y experiencias se están también reduciendo las posibilidades de mantener las situaciones gratificantes y las condiciones valiosas de lo que, hasta entonces, era su vida. En diferentes estudios sobre la calidad de vida del enfermo oncológico se ha observado que, durante la fase de tratamiento, las actividades cotidianas en las que un mayor número de enfermos oncológicos indican cambios son las domésticas, las laborales y las de ocio. En particular, los cambios en las relaciones con los amigos y en el ocio se han relacionado consistentemente con un estado de ánimo deprimido, valoraciones negativas de la calidad de vida, cansancio, insomnio y dolor ${ }^{(3-5)}$. Al concluir el tratamiento, aunque se observa una recuperación paulatina de la mayoría de las actividades cotidianas, es siempre menor entre los pacientes con un estado depresivo más acentuado ${ }^{(6,7)}$.

La progresiva pérdida de funcionalidad, como resultado de la evitación de experiencias dolorosas (miedos, pérdidas,...), además de apartar a las personas de las situaciones cotidianas que le proporcionarían bienestar, las mantiene enganchadas a las condiciones aversivas que pretenden evitar. Cuanto mayor sea el esfuerzo por controlar/evitar una situación, mayor presencia cobra. De esta manera y en contra de lo que se pretendía, el malestar crece. Esta psicopatologización de la enfermedad, podría ayudar a explicar las alteraciones emocionales y el deterioro de la calidad de vida del enfermo oncológico.

Desde una perspectiva contextual, se sugiere que los pacientes oncológicos se 
beneficiarían de una intervención basada en la Activación Conductual para mejorar el afrontamiento a la enfermedad y prevenir alteraciones emocionales. Se trataría de lograr el compromiso del sujeto con el curso de su vida, involucrándose en aquellas actividades capaces de activar sus fuentes de reforzamiento cotidianas (activación), de mantener conductas saludables y eliminar/reducir conductas de enfermedad.

Este trabajo evalúa la eficacia de la Activación Conductual con pacientes con cáncer de pulmón. La intervención se diseñó conforme a los principios y procedimientos de la Terapia de Activación ${ }^{(8-11)}$. La utilidad de este tratamiento con enfermos oncológicos y depresivos ha sido probada en diferentes estudios ${ }^{(12-14)}$. Sin embargo, a excepción de los trabajos de nuestro grupo (15) no se conocen otros estudios que trabajen con pacientes oncológicos de reciente diagnóstico con el objetivo de promocionar la calidad de vida durante el tratamiento con quimioterapia.

\section{MÉTODO}

\section{Participantes}

Se seleccionaron de forma consecutiva todos los pacientes con cáncer de pulmón que acudían por primera vez a la Unidad de Oncología Médica del Hospital de Cabueñes (SESPA, Gijón. España), independientemente del estadio inicial de la enfermedad y siempre y cuando el tratamiento oncológico a seguir fuera la quimioterapia en cualquiera de sus modalidades (adyuvante, neoadyuvante, de inducción). Al final de la primera consulta, el oncólogo solicitaba a cada paciente su colaboración para participar en un estudio controlado sobre: (a) la utilidad de una intervención psicológica para mejorar la calidad de vida (Grupo Experimental -G.E.-); (b) la evaluación de la calidad de vida del enfermo oncológico (Grupo Control -G.C.-). De forma aleatoria (según tabla de números) se estableció la asignación experimental. El total de participantes fueron 90 (G.C.: $N=40$ / G.E.: $N=50$ ). Aunque ninguno de los pacientes rechazó inicialmente participar en el estudio, fueron elevadas las tasas de abandono obligado por fallecimiento (G.C.: $N=10$ / G.E.: $N=13$ ) o deterioro físico con hospitalización (G.C.: N=14 / G.E.: $N=14), 7$ rechazaron participar en el G.C. y 9 en el G.E.

El G.C. se compuso por 40 pacientes, 36 hombres $(90 \%)$ y 4 mujeres $(10 \%)$, cuya edad media era 60,92 años (DT=8,33; $M_{n}=44 ; M_{x}=78 ; R=34$ años). La mayoría de los pacientes estaban casados o viviendo en pareja $(77,5 \%)$, tenían hijos $(77,5 \%)$ y vivían con algún familiar (92,5\%). Sólo un $5 \%$ de los pacientes no tenían estudios y la mayoría poseía estudios primarios $(62,5 \%)$. Con respecto a la situación laboral, un $55 \%$ estaba jubilado, un $30 \%$ con baja laboral y sólo un 5\% se hallaban en activo. El resto eran amas de casa $(7,5 \%)$ o estaban en paro $(2,5 \%)$.

El G.E., lo compusieron 50 pacientes, 37 hombres $(74 \%)$ y 13 mujeres $(26 \%)$ con una edad media de 61,64 años (DT=9,57; $M_{n}=43 ; M_{x}=78 ; R=35$ años). La mayoría de los sujetos estaban casados o viviendo en pareja $(78 \%)$, tenían hijos $(92 \%)$ y vivían con algún familiar (95,91\%). Un 8,3\% no tenía estudios y, del resto, la mayoría contaba con estudios primarios (56,3\%). En cuanto a la situación laboral, un $54 \%$ estaban jubilados, un $36 \%$ de baja, un $4 \%$ permanecía en activo y un $6 \%$, eran amas de casa.

Con respecto al tipo de tumor, en el G.C., el $75 \%$ de los pacientes presentaban un tumor no microcítico, siendo los más numerosos del subtipo epidermoide $(63,3 \%)$ y el adenocarcinoma (30\%). En cuanto a los estadios, los mayores porcentajes eran para los estadios IV y III-B (ambos 33,3\%) y III-A $(30 \%)$. Dentro de los tumores microcíticos, el $70 \%$ presentaban enfermedad limitada y $30 \%$ enfermedad extensa. En el G.E., el 
$74 \%$ de los pacientes presentaban un tumor no microcítico, del subtipo adenocarcinoma $(48,6 \%)$ y epidermoide $(40,5 \%)$. El $37,8 \%$ de los participantes se encontraba en un estadio IV; $32,4 \%$ en III-B y $18,9 \%$ en III-A. Dentro de los tumores microcíticos, el $75 \%$ presentan enfermedad limitada y el $25 \%$ enfermedad extensa. Respecto al tratamiento de quimioterapia los pacientes con tumores no microcíticos, en ambos grupos, la mayoría recibió Cisplatino + Gemcitabina $(78,4 \%)$. Los pacientes con tumores del tipo microcítico recibieron principalmente Carboplatino + VP16 (50\%), seguido de Genoxal + Adriamicina + VP16 (33,3\%).

No existen diferencias estadísticamente significativas entre grupos ni en las variables sociodemográficas ni clínicas y, aunque el número de pacientes en cada grupo es distinto, la prueba estadística Binomial indica que las proporciones observadas del total de la muestra en cada uno de los grupos no es diferente del 50\% $(p=0,343)$.

\section{Instrumentos}

- Protocolo clínico (ad hoc). Cumplimentado en la primera consulta por el oncólogo y psicólogo. Incluye datos clínicos y socio-demográficos.

- Escala de Karnofsky ${ }^{(16)}$. Trata de cuantificar el estado general del paciente oncológico, reflejando el grado de autonomía en un rango de 0 (-) a 100 (+). Cuenta con garantías psicométricas y se considera un indicador válido del estado físico general que correlaciona significativamente con diversas variables físicas y psicológicas ${ }^{(17)}$. Fue cumplimentada por el oncólogo en todas las sesiones.

- Escala de ansiedad y depresión hospitalaria $(\mathrm{HAD})^{(18)}$. Esta escala evalúa el estado emocional de los sujetos que son atendidos en servicios hospitalarios de consulta externa no psiquiátricos, excluyendo cualquier referencia a síntomas físicos. Es una escala formada por 14 ítems que se dividen en dos subescalas, ansiedad y depresión, cada una formada por 7 ítems. En cada una de las escalas, valores totales entre 0 y 7 indican ausencia de caso clínico, entre 8 y 10 caso dudoso y de 11 a 21, caso clínico. Las propiedades psicométricas de esta escala parecen ser adecuadas, mostrando una fiabilidad de 0,80 para cada una de las subescalas y una validez de $0,70^{(19)}$. Se empleó en todos los momentos de evaluación.

- Cuestionario de calidad de vida de la EORTC QLQ-C30 (20). Contiene una escala global de calidad de vida, cinco escalas funcionales (funcionamiento físico, rol, emocional, social y cognitivo), tres escalas de síntomas (fatiga, dolor, y náuseas/vómitos) e ítems individuales que evalúan síntomas adicionales. En las escalas funcionales las puntuaciones más altas indican mejor funcionamiento y a la inversa en las escalas de síntomas. Diferentes estudios prueban las garantías psicométricas del instrumento $^{(20)}$. En el estudio de validación con población española de Arrarás ${ }^{(21)}$, los análisis factoriales confirman la estructura de la mayoría de las escalas. Las escalas RF y SF alcanzan un $\alpha$ de Cronbach $>0,60$, aunque el resto presentan coeficientes de fiabilidad adecuados y la consistencia interna global es de $\alpha=0,78$. Se empleó a partir del segundo ciclo de quimioterapia, en todos los momentos de medida.

- Entrevista semiestructurada ${ }^{(22)}$. Diseñada para recoger información acerca de los síntomas de la enfermedad y tratamiento, estado emocional y funcionamiento del paciente en los distintos ámbitos de la vida cotidiana. Se empleó en todas las sesiones, en ambos grupos. En el G.E., en el contexto de la entrevista, se analizaban en términos contextuales los síntomas, las rutinas cotidianas y las emociones y pensamientos del paciente sobre la enfermedad y tratamiento. En el G.C. no se prestaba ninguna atención a las competencias/déficit de los sujetos en relación al afrontamiento de las situaciones referidas. 
- Escala de Estado General (EG) ${ }^{(22)}$. Escala analógica que permite puntuar de 0 (-) a 10 (+) estado general percibido por el paciente en el periodo comprendido entre sesiones. Utilizada en cada sesión.

- Escala de Estado de Salud (SP)(22). Escala analógica que permite puntuar de 0 (-) a 10 (+) el estado de salud percibido por el paciente en el periodo comprendido entre sesiones. Utilizada en cada sesión.

\section{Procedimiento}

El proceder con el grupo experimental y control se integró en la rutina de consultas y sesiones de tratamiento de quimioterapia establecidas por los oncólogos. Todos los pacientes fueron atendidos individualmente durante 45 minutos por una psicóloga después de la consulta médica y antes de recibir un ciclo de quimioterapia. El protocolo de tratamiento médico consistió en una pauta de cuatro ciclos de quimioterapia. A todos los pacientes se les realizó un seguimiento trimestral después de finalizado el tratamiento.

En el grupo control se siguió en todas las sesiones un mismo procedimiento. Durante la sesión, el paciente cumplimentaba los instrumentos de evaluación estandarizados y describía su situación personal en cada uno de los ámbitos examinados ${ }^{(22)}$. Durante las sesiones, en ningún momento, se prestó atención diferencial a los déficits o competencias de los pacientes en relación al afrontamiento de su situación actual, ni se les proporcionó entrenamiento en ningún procedimiento psicológico.

En el grupo experimental el procedimiento se estructuró para proporcionar una explicación contextual-funcional de los problemas/limitaciones del paciente oncológico y para activar a la persona en la dirección de: (a) mantener/restablecer actividades relevantes y rutinas cotidianas; (b) incrementar actividades gratificantes; (c) eliminar conductas de enfermedad; (d) mo- dificar el patrón de evitación experiencial. En el contexto de la entrevista, el terapeuta enseñaba al paciente a analizar en términos contextuales su conducta. En concreto: rutinas cotidianas, síntomas y limitaciones físicas; emociones y pensamientos/preocupaciones (sobre la enfermedad y tratamiento). Se perseguía que la persona aprendiese a observar la relación entre lo que hace, siente, piensa y lo que ocurre en su entorno. $Y$, en consecuencia, a identificar los eventos/condiciones que mantienen $\mathrm{y} / \mathrm{o}$ debilitan comportamientos concretos. Se analizaban tanto las conductas saludables como las conductas de enfermedad y de evitación (de pensamientos/emociones en relación a la enfermedad y tratamiento oncológico). El objetivo último era establecer, con el paciente (y allegados), un compromiso activo para fomentar sus propias competencias/habilidades y mantener las actividades relevantes y gratificantes de su vida.

El proceso de las sesiones, de acuerdo a la lógica de la Activación Conductual, se ajustó siempre al caso particular. El análisis funcional de la conducta constituyó el procedimiento fundamental para determinar las metas y tareas de la intervención para cada caso y en cada sesión. Se estructuraron y programaron tareas para casa encaminadas al mantenimiento de rutinas cotidianas gratificantes y que tuviesen una alta probabilidad de reforzamiento en el entorno personal y familiar del paciente. Se buscaba movilizar los propios recursos del paciente y también provocar un cambio de contingencias en el entorno. Así, durante la sesión, se solicitaba al paciente/ allegados que propusiesen posibles soluciones a sus problemas/limitaciones. Sólo cuando se carecía de repertorios adecuados, se entrenaban habilidades específicas. Se comprobaba que la persona no sólo supiese qué hacer y cómo, sino cuándo y dónde o en qué condiciones actuar. Es decir, que además de "saber qué hacer" supiese reconocer las "contingencias" 
que con alta probabilidad fortalecerían conductas saludables y relevantes para la persona. Entonces, se solicitaba su compromiso para actuar (a pesar de la enfermedad o el estado de ánimo) y observar los resultados. En cada sesión, la psicóloga y el paciente revisaban el cumplimiento y resultados de las tareas y se analizaba la función que desempeñaban las actividades para la mejora de su calidad de vida y estado emocional.

Entre las técnicas más empleadas se destacan: la autoobservación y registro, el ensayo y modelado de conducta, la elaboración de jerarquías de actividad, la programación de conductas y el manejo de contingencias, la resolución de problemas, el entrenamiento en habilidades sociales, el uso de metáforas y ejercicios experienciales para facilitar la aceptación y distanciamiento de emociones y pensamientos. El protocolo de objetivos y tareas para cada sesión se expone de manera detallada en Fernández et al. ${ }^{(15)}$.

\section{Análisis de datos}

Se obtuvieron los estadísticos descriptivos correspondientes en el grupo control y experimental para cada variable y momento de evaluación. Todas las variables cuantitativas cumplían el criterio de normalidad.

Se empleó un diseño de medidas parcialmente repetidas con una variable entre sujetos, con dos niveles (G.C. y G.E.) y una variable intra-sujetos, con 5 niveles (4 medidas de tratamiento y un seguimiento). El estudio de la evolución y tendencia de las variables se ha realizado mediante (a) el Modelo Lineal General (MLG) con los pacientes de los que se dispone de todos los datos; y el Modelo Lineal Mixto (MLM) dada la pérdida de muestra a lo largo de las sesiones. En el MLG se tuvo en cuenta la presencia o ausencia de esfericidad y se corrigieron los grados de libertad cuando fue necesario de acuerdo a Greenhouse y Geisser o a Huynh y Feldt ${ }^{(23)}$. Se examinó la evolución de las variables mediante contrastes polinómicos. Y se observó el tamaño del efecto mediante $\eta^{2}$ y la potencia de prueba a posteriori. Se utilizó el MLM con todos los participantes porque permite realizar el análisis de las medidas repetidas cuando se tienen datos perdidos y analizar la estructura de covarianza que subyace a los datos. Se han realizado comparaciones post hoc por pares controlando la tasa de error mediante Bonferroni.

El análisis de los datos se llevó a cabo con el paquete estadístico SPSS.19 y el nivel de significación se ha establecido a priori en 0,05.

\section{RESULTADOS}

- Estado General (EG). En la tabla 1 se advierte que, conforme avanzan las sesiones, el estado general apenas varía en el G.C. y mejora en el G.E. (medias y percentiles más elevados y menor variabilidad en la medida). Los resultados del MLG y MLM indican que, a lo largo del tiempo, las valoraciones mejoran. El MLG señala diferencias estadísticamente significativas entre los grupos $(p=0,02)$ a favor del G.E. No obstante, se percibe una tendencia cuadrática de los datos $(p=0,009)$. De hecho, en el G.E., en el primer seguimiento, la valoración empeora.

- Estado de Salud (ES). Los resultados del MLG y MLM muestran diferencias estadísticamente significativas en la percepción de la salud conforme avanzan las sesiones en la variable tiempo. Las medidas señalan la mejoría del G.E. con percentiles más elevados y menores desviaciones típicas. Por el contrario, el G.C. mantiene o empeora la percepción de su salud. Esta interacción entre las variables grupos y tiempo no se observa estadísticamente, quizás por falta de potencia de prueba debido a la pérdida de muestra según avanzan las sesiones (ver la tabla 1). 
- Índice Karnofsky (IK): La valoración médica del IK se halla en torno a 85/86 en ambos grupos, con un leve descenso a lo largo del tiempo. La valoración es mejor para los pacientes del G.E. (también con menor desviación típica). Ni el MLM ni el MLG detectan diferencias entre los grupos, aunque el MLM, sí aprecia este cambio a través del tiempo $(p=0,000)$ (ver la tabla 1$)$.

- Ansiedad (HAD-A) y Depresión (HAD$D)$ : En la tabla 1 se observa como las puntuaciones medias de ambos grupos no indican sintomatología ansiosa y depresiva con significación clínica (puntuaciones medias $<7$ ). En ambas variables y a lo largo del tiempo, sólo el G.E. parece experimentar mejoría al presentar un descenso paulatino de las medias y desviaciones. Aunque el MLG no capta este aspecto, debido quizás al desgaste paulatino de la muestra, el MLM sí detecta las diferencias entre grupos.

- Estatus Global de Calidad de Vida (QLQ-C-30.QoL): Ambos grupos presentan puntuaciones muy similares (entre 60 y 70). El G.E. experimenta mayor calidad de vida en los seguimientos, con percentiles más bajos y menos variabilidad en las medidas. Los resultados del MLG y MLM no muestran diferencias estadísticamente significativas en ninguna de las fuentes de variación (ver la tabla 1).

- Funcionamiento físico (QLQ-C-30. $P F)$ : Los estadísticos descriptivos muestran cómo, a partir de la tercera sesión, el G.C. empeora y prácticamente se mantiene así hasta el final. Por el contrario, el G.E. experimenta una mejoría muy clara, aunque progresivamente va empeorando. Esta interacción entre ambos grupos, aunque tímidamente, resulta estadísticamente significativa en el MLG (tendencia cuadrática, $p=0,078$ ) (tabla 2).

- Funcionamiento Emocional (QLQC-30.EF): En el G.C. se observa un empeoramiento inicial con tendencia a recuperarse en el seguimiento. En el G.E. ocurre lo contrario, hay una mejoría inicial con tendencia a empeorar en los últimos seguimientos. De los análisis realizados únicamente el MLM aprecia diferencias estadísticamente significativas entre ambos grupos $(p=0,06)$ (Tabla 2$)$.

- Funcionamiento Cognitivo (QLQ-C30CF): En la Tabla 2 se observa como el G.E. mejora en el tiempo, mientras que las puntuaciones del G.C. se mantienen y empeoran en el seguimiento. Esta interacción entre las variables tiempo y grupos la detecta, tanto el MLG $(p=0,033)$ mostrando una tendencia Lineal de los datos $(p=0,021)$, como el MLM, aunque con una menor significación estadística $(p=0,09)$.

- Funcionamiento de Rol (QLQ-C-30. RF). En el G.E. se observa durante la intervención un mejor funcionamiento de Rol y, aunque se deteriora levemente en el primer seguimiento, se recupera en los siguientes. El G.C. muestra peores puntuaciones, pero mejora en los seguimientos. El MLG detecta estos cambios a través del tiempo y una tendencia Lineal significativa $\left(p=0,034 ; \eta^{2}=0,450\right)$. El MLM no destaca ninguna diferencia estadísticamente significativa (ver la tabla 2).

- Funcionamiento Social (QLQ-C-30. $S F)$. Las puntuaciones que se recogen en la tabla 2 sugieren que el tiempo favorece en ambos grupos el funcionamiento social. Pero es el G.E. el que más se destaca, con medias más altas y desviaciones típicas inferiores a las del G.C. El MLG capta este aspecto y una tendencia Lineal significativa $(p=0,032)$.

- Escala QLQ-LC-13: Durante el tratamiento con quimioterapia los pacientes de ambos grupos parecen tener un adecuado control de los síntomas de la enfermedad y tratamiento. Los datos sugieren que la mayoría de los síntomas están más presentes en el G.C. y que se mantienen en el tiempo, mientras que el G.E. mejora a lo largo de las sesiones y sólo empeora en el seguimiento (ver la tabla 3). Las diferencias entre grupos y la interacción se captan, 


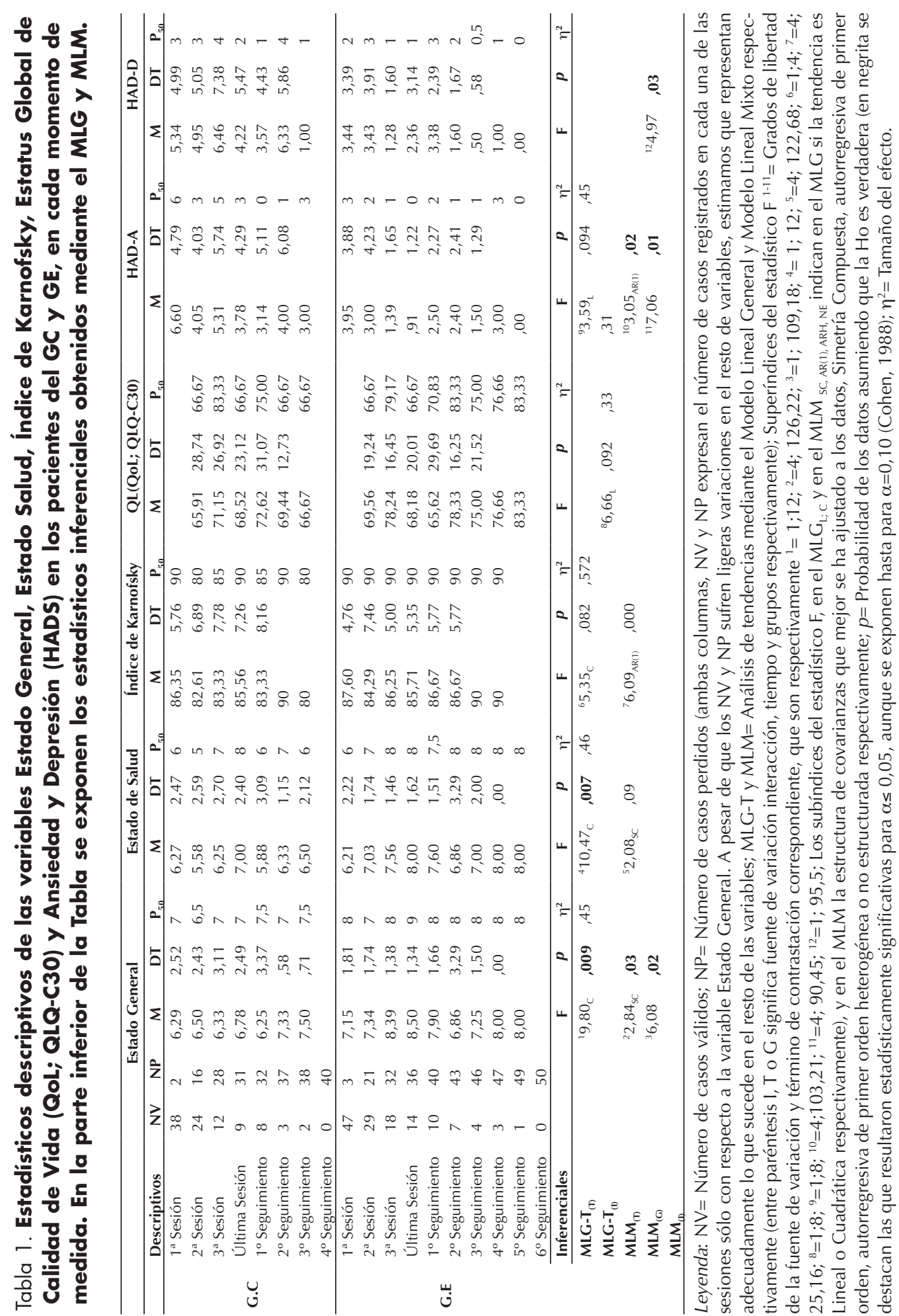




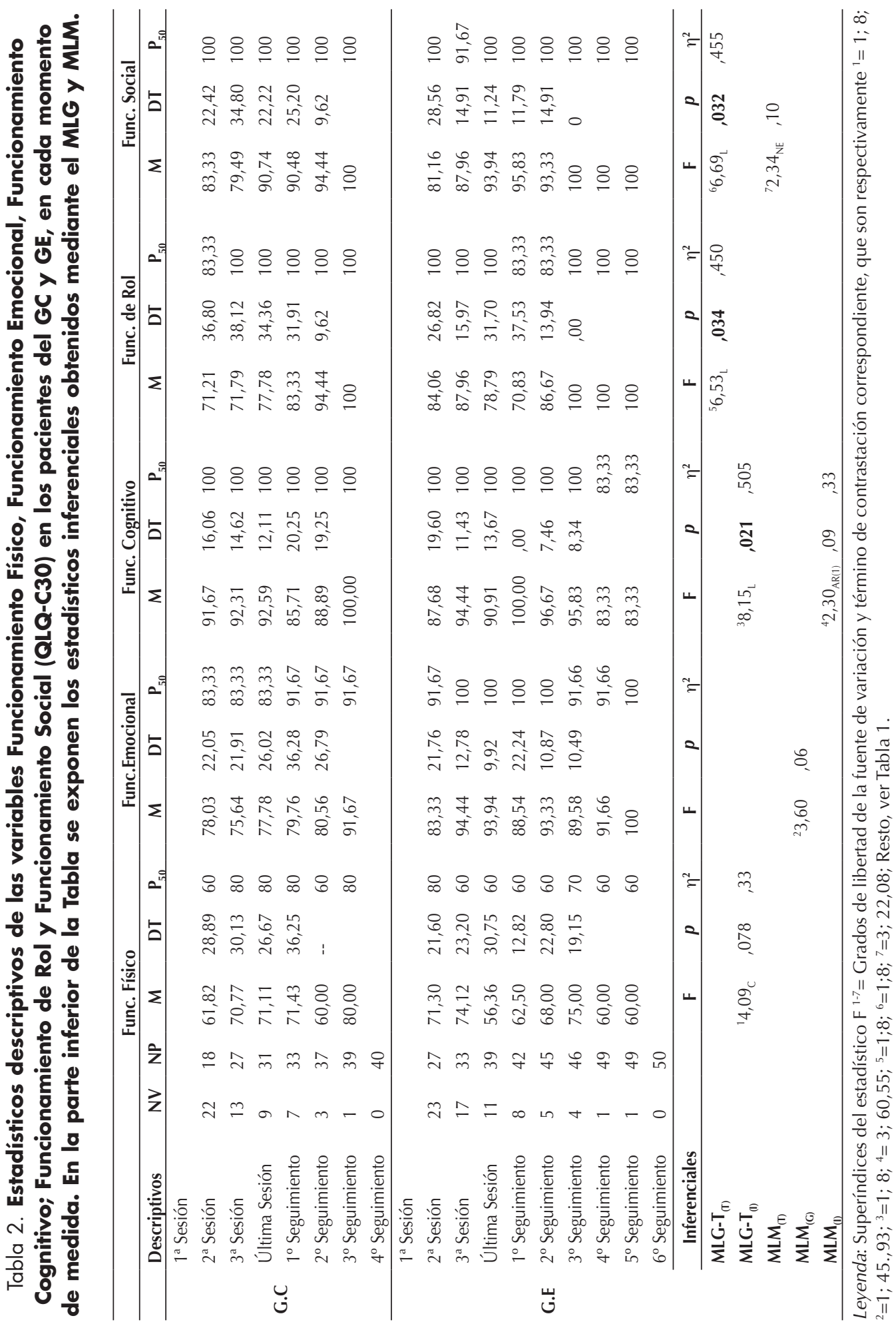




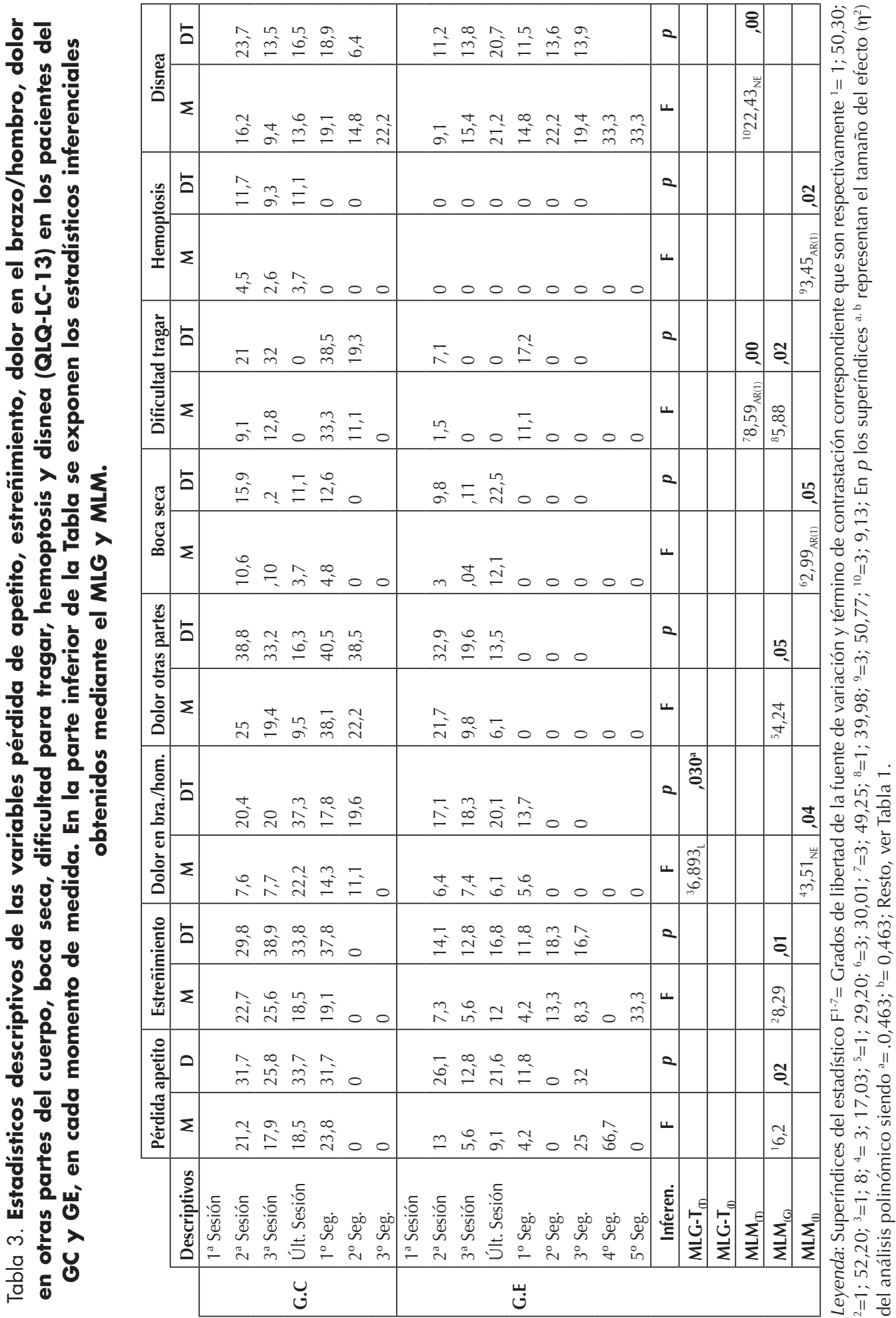


dada la escasa presencia de los síntomas y pérdida de sujetos, a través del MLM e indican, en todos los casos, una mejor situación del G.E., en concreto, respecto al apetito, estreñimiento, dolor en el brazo y otras partes del cuerpo, boca seca y dificultad para tragar, hemoptisis, disnea.

\section{DISCUSIÓN}

Este trabajo ha tenido como objetivo analizar el efecto de una intervención psicológica basada en la Activación Conductual sobre la calidad de vida y el estado emocional de pacientes con cáncer de pulmón durante el tratamiento con quimioterapia.

La intervención experimental se diseñó conforme a los principios y técnicas fundamentales de la Activación Conductual(8-11). Desde la explicación y abordaje contextual-funcional de los problemas/ limitaciones del paciente oncológico, el procedimiento se estructuró para buscar un compromiso activo de la persona con las actividades relevantes y gratificantes de su vida, aún a pesar de la enfermedad.

Las condiciones que definen la calidad de vida del enfermo oncológico (síntomas, estado emocional y funcionalidad) se valoraron mediante autoinforme y cuestionarios estandarizados en cada sesión de tratamiento y en seguimiento trimestral. Los efectos de la intervención experimental se midieron en relación a un G.C. en el que se empleó un mismo número de sesiones de igual duración que se dedicaron, exclusivamente, a la evaluación de la calidad de vida. Este grupo, en tanto que no recibió instrucción, ni entrenamiento en ningún procedimiento psicológico específico, ni atención diferencial respecto a sus estrategias de afrontamiento, se consideró idóneo para descartar que los resultados del G.E. pudieran atribuirse a la mera atención dispensada. Además, y en cuanto que supuso para el paciente la oportunidad de expre- sar sus preocupaciones y emociones a una audiencia especializada, pudo tener un valor terapéutico, aunque inespecífico. En consecuencia, este procedimiento no sólo ofrece garantías éticas como grupo control sino que proporcionaría un valor añadido a los resultados del grupo de Activación Conductual.

Los datos clínicos y sociodemográficos de los participantes permiten considerar que son representativos de la población con cáncer de pulmón y, por tanto, generalizables. Son mayoritariamente hombres $(81,1 \%)$, con una edad medida en torno a los 62 años. Por el tipo de tumor, un $72,22 \%$ presenta un carcinoma no microcítico, que supone el $80 \%$ de los cánceres de pulmón en la población general. En cuanto al estadio de la enfermedad, se observa como la mayoría de los pacientes, en el momento del diagnóstico, se hallan en estadios avanzados, ajustándose a lo observado en la población ${ }^{(24)}$. En cuanto a los grupos (G.C. / G.E.), representan las mismas características clínicas y sociodemográficas de la muestra total.

Antes de la intervención, ambos grupos, eran equiparables en todas las variables analizadas (sociodemográficas, clínicas, IK, estado de salud y calidad de vida percibida y puntuaciones en las subescalas del HAD y del QLQ-C30), lo que permite evaluar fiablemente los efectos de la intervención y del transcurso del tiempo. Por lo demás, el tamaño inicial de los grupos (G.C.: $N=40 ; \quad$ G.E.: $N=50$ ) es suficiente para garantizar un adecuado control estadístico de los datos. En consecuencia, puede asumirse la generalización de los resultados de este estudio. No obstante, la destacada pérdida de muestra por fallecimiento/hospitalización, aunque responda a la evolución de la enfermedad, sí puede limitar la potencia de los resultados.

La evolución y tendencia del estado general y de salud percibida indican que, a lo largo del tiempo, ambas condiciones 
mejoran. Aún así y a pesar de la pérdida de muestra por fallecimientos y hospitalizaciones, se encuentran diferencias significativas entre grupos a favor del G.E., en particular, en la percepción del estado general. La mejoría del G.E. durante el tratamiento se pone en relación a los cambios comportamentales provocados por la Activación Conductual.

Ahora bien, también se observa que, concluida la intervención, la valoración del estado general de los participantes parece empeorar. Esta circunstancia, aunque podría cuestionar la capacidad de la intervención para mantener los efectos a medio plazo, no habría de comprometer la especificidad de la misma para provocar el cambio. Ha de tenerse en cuenta que, antes de la intervención, el G.E. y el G.C. eran equiparables clínica y sociodemográficamente y que el procedimiento utilizado en el G.C. reduce la posibilidad de atribuir las diferencias entre grupos a factores inespecíficos. En todo caso, aún cuando la Activación Conductual resultase útil para mejorar la calidad de vida percibida, también parece, tal y como se aplicó en este estudio, insuficiente para mantener la mejoría a medio plazo. Las modalidades breves de la terapia de Activación Conductual se han aplicado con éxito, aunque siempre han empleado un número de sesiones superior al utilizado en este trabajo, entre 6 y $12^{(25,26)}$. Otros estudios de carácter descriptivo que han observado una evolución favorable del estado general y de salud percibida durante el tratamiento oncológico, la han atribuido a la favorable repercusión emocional asociada a la sensación de control sobre la enfermedad que proporciona el tratamiento $y$, por supuesto, a los propios efectos del mismo para aliviar los síntomas ${ }^{(27)}$. Aunque estas condiciones modulen nuestros resultados y, así se contemplan, cabría esperar que afectasen por igual a ambos grupos. Pero, precisamente, dada la desigual evolución de ambos grupos durante el tratamiento, parece justificado atribuir a la Activación Conductual la mejoría del G.E.

La calidad de vida evaluada por el médico mediante el IK, en ambos grupos, es relativamente alta y más elevada en el G.E. Y, aunque los cambios en el tiempo y entre grupos no alcanzan la significación estadística, sí se observa una tendencia a empeorar a medida que avanzan las sesiones y a mejorar en los seguimientos. Esta valoración del médico parece contraria a las valoraciones realizadas por el paciente. Las discrepancias entre las distintas fuentes de información en la valoración de la calidad de vida es un hecho reiteradamente constatado. En general, las valoraciones del personal sanitario muestran una alta correlación con los parámetros físicos y son menos sensibles a los cambios percibidos por el paciente en su salud, en su rutina y en su estado general. En este sentido, parece que existe mayor acuerdo entre los médicos y los pacientes en relación a aspectos físicos y menor respecto a los emocionales ${ }^{(28-31)}$.

El estado físico y síntomas de la enfermedad y tratamiento es un ámbito importante de la calidad de vida del paciente oncológico y, no cabe duda, que modula el grado de funcionalidad del paciente en otros ámbitos de la vida cotidiana. En conjunto y evaluados mediante el QLQ-C30, entre los participantes se observó la escasa relevancia clínica de algunos síntomas como las alteraciones gastrointestinales, náuseas y vómitos, muy probablemente, debido al tipo y pauta de quimioterapia administrada. Los síntomas con mayor presencia fueron el cansancio, el dolor, la pérdida de apetito y la dificultad para dormir, como también se ha puesto de manifiesto en otros estudios ${ }^{(32)}$. La mayoría de los síntomas están más presentes en el G.C. y también se mantienen en el tiempo. Por el contrario, el G.E. mejora a partir de la segunda sesión de intervención 
y sólo empeora en el primer seguimiento. A pesar de la escasa presencia de los síntomas y de la pérdida de sujetos a lo largo de las sesiones, las diferencias observadas entre grupos y la evolución y tendencia de la sintomatología mostraron una mejor situación en el G.E. La influencia de la Activación Conductual en la mejora de la sintomatología del G.E., como en el caso de los digestivos, apetito o dolor, evidentemente, sólo puede explicarse como un efecto del cambio del comportamiento de los pacientes en relación a la enfermedad. Los síntomas que mejor evolución han mostrado en el grupo de Activación Conductual, en otros estudios, ya se han relacionado con cambios en las rutinas de los pacientes y en el estado emocional ${ }^{33-}$ ${ }^{35)}$. Lo que sugeriría, nuevamente, la adecuación del procedimiento experimental para reducir conductas de enfermedad e incrementar actividades relevantes y gratificantes de las que depende el bienestar diario de los pacientes.

El funcionamiento en diferentes ámbitos de la vida cotidiana, evaluado mediante las escalas de rol, social y cognitivo del QLQ-C30, indicó siempre mayor competencia en el G.E. Y sólo se observó una tendencia a mejorar de forma lineal estadísticamente significativa con la Activación Conductual. En particular, en la tercera sesión, se observa un marcado mejor funcionamiento en el G.E. El G.C. parece mantener el mismo nivel de funcionamiento a lo largo de todas las sesiones. Los cambios en la funcionalidad de los pacientes del G.E. son la mejor constatación de la utilidad de la intervención para promover la calidad de vida durante el tratamiento oncológico y, parece razonable, atribuirlo al efecto de la Activación Conductual para promover el mantenimiento/incremento de actividades relevantes y gratificantes de la vida cotidiana.

La adaptación psicológica de los pacientes durante el tratamiento oncológico, en general, era buena. Las puntuaciones medias obtenidas con el HAD no indicaban la presencia de patología ansiosa o depresiva en ninguno de los dos grupos. Resultados similares se han obtenido en estudios previos y por otros investigadores $^{(36-37)}$. No obstante, en otros trabajos sí se han constatado porcentajes más elevados de pacientes con alteraciones emocionales desde el diagnóstico oncológico ${ }^{(38,39)}$. Estas discrepancias, con frecuencia, se explican por las diferencias clínicas y sociodemográficas entre los participantes y entre los procedimientos e instrumentos de evaluación empleados ${ }^{(40)}$. En nuestro estudio, asumiendo que ambos grupos pueden considerarse representativos clínica y sociodemográficamente de la población diana y que los instrumentos de evaluación cuentan con las debidas garantías, se constató que los pacientes del G.E. mostraron una tendencia a mejorar significativamente la sintomatología ansiosa y depresiva hacia el final del tratamiento. Incluso, cuando cabría esperar que fuesen poco susceptibles de cambio, precisamente, por la ausencia de patología clínica (HAD). Con la subescala de Funcionamiento Emocional del QLQ-C30, también se observó un mejor estado emocional en el G.E., aunque con una tendencia a empeorar en los seguimientos. Ambos resultados avalan la eficacia de la AC, por otra parte ya documentada para el tratamiento de la depresión con pacientes oncológicos ${ }^{(12-14)}$.

En definitiva, se sugiere que la mejoría emocional y en el resto de los ámbitos de la calidad de vida del G.E. puede atribuirse a la Activación Conductual. Dadas las peculiaridades del G.C. puede afirmarse que los cambios en el G.E. no son la mera consecuencia de la atención dispensada y, en consecuencia, el procedimiento experimental se puede considerar específico para provocar el cambio. Ahora bien, los datos del seguimiento también señalan cierta pérdida de funcionalidad de los pacientes 
del G.E., lo que sugeriría la inadecuación de la intervención para mantenerlo. En este estudio, en un afán por mejorar la eficiencia de la intervención, muy probablemente se comprometió su efectividad. Las cuatro sesiones que se invirtieron con cada paciente, aunque permitieron integrar en una misma visita hospitalaria la intervención psicológica y médica, probablemente, resultaron insuficientes para consolidar, en pacientes y allegados, las competencias necesarias para reconocer y movilizar las contingencias privadas y del entorno de las que puede depender el mantenimiento de las actividades gratificantes de su vida cotidiana. Condiciones centrales de la Activación Conductual, como son la monitorización programada de las actividades relevantes y de las contingencias de reforzamiento o el reforzamiento en terapia de conductas saludables, se entiende que se debilitaron al finalizar la intervención, con la consiguiente pérdida de eficacia. Pero también debe destacarse que, a medida que transcurría el tiempo y dada la condición clínica de los participantes, el deterioro progresivo de la enfermedad comprometió abiertamente la capacidad de las personas para mantener las actividades relevantes de la vida, al tiempo que fue la principal condición responsable de los abandonos. El estado físico de los participantes, en conjunto, estaba deteriorado y prueba de ello fue la alta tasa de abandono por deterioro físico con hospitalización y éxitus. Dada esta situación, el hecho de que sólo el G.E. haya tenido una evolución favorable en todas las escalas de funcionalidad, confirmaría que, a pesar de la enfermedad, la recuperación de las rutinas cotidianas fue un objetivo valioso para los participantes y relevante para el paciente oncológico. Adicionalmente, la mejoría atribuible al tratamiento médico también pudo actuar facilitando la recuperación de las actividades. En cualquier caso, todos los participantes recibían trata- mientos quimioterapéuticos similares pero sólo en el grupo de Activación Conductual se produjeron cambios significativos en la funcionalidad y estado emocional de las personas. Aunque, el principal ingrediente responsable de los cambios de los pacientes del G.E. se pone en el cambio conductual, cuáles son los componentes de la intervención necesarios para provocar y mantener tales cambios es una cuestión que ha de ser investigada.

En definitiva, se sugiere que este estudio aporta evidencia suficiente acerca del interés de los objetivos y la especificidad de los procedimientos de una intervención centrada en la Activación Conductual durante el tratamiento oncológico para promover la calidad de vida y mejorar el estado emocional. No obstante, esta es una línea de investigación abierta que tiene pendiente indagar qué características de los pacientes harían idónea esta intervención durante el tratamiento oncológico y, alternativamente, qué peculiaridades de la misma (duración, momento de inicio, componentes...) mejorarían la eficacia y efectividad con los pacientes de cáncer de pulmón y con otros tipos de cáncer.

\section{Agradecimientos}

Este trabajo ha sido financiado por el Ministerio de Ciencia e Innovación en el marco I+D+l (Ref.: PSI2009-09601).

\section{REFERENCIAS BIBLIOGRÁFICAS}

1. Hughes $S$, Jaremka LM, Alfano CM, Glaser R, Povoski SP, Lipari AM, et al. Social support predicts inflammation, pain, and depressive symptoms: Longitudinal relationships among breast cancer survivors. Psychoneuroendocrinology 2014;42:3844. Doi: 10.1016/j.psyneuen.2013.12.016

2. Luszczynska A, Pawlowska I, Cieslak R, Knoll N, Scholz U. Social support and quality of life among lung cancer patients: 
A systematic review. Psychooncology 2012;22:2160-8. Doi: 10.1002/pon.3218

3. Espantoso R, Fernández C, Padierna C, Amigo I, Villoria E, Gracia JM, et al. Calidad de vida en pacientes oncológicos un año después de finalizado el tratamiento. Psicooncología 2007;4:43-57.

4. Fernández C, Padierna C, Villoria E, Amigo I, Fernández R, Peláez I. Repercusión de la ansiedad y depresión en el estado físico y funcionalidad de enfermos oncológicos durante el tratamiento con quimioterapia. Psicothema 2011;23:374-81.

5. Solberg N, Liu H, Patten CA, Rausch SM, Sloan JA, Garces YI, et al. Physical activity level and quality of life in long term lung cancer survivors. Lung Cancer 2012;77:611-6. Doi: 10.1016/j. lunG.C.an.2012.05.096.

6. Boyes AW, Girgis A, D'Este CA, Zucca AC, Lecathelinais C, Carey ML. Prevalence and predictors of the shortterm trajectory of anxiety and depression in the first year after a cancer diagnosis: A population-based longitudinal study. J Clin Oncol 2013;31:2724-9. Doi: 10.1200/ JCO.2012.44.7540

7. Cataldo JK, Brodsky JL. Lung cancer stigma, anxiety, depression and symptom severity. Oncology 2013;85:33-40. Doi: $10.1159 / 000350834$

8. Hopko DR, Bell JL, Armento MEA, Hunt MK, Lejuez CW. Behavior therapy for depressed cancer patients in primary care. Psycotherapy 2005; 42:236-43.

9. Jacobson NS. Can contextualism help? Behav Ther 1997;28:435-43. Doi: 10.1016/S0005-7894(97)80092-5

10. Jacobson NS, Martell CR, Dimidjian S. Behavioral activation treatment for depression: returning to contextual roots. Clin Psychol 2001;8:255-70. Doi: 10.1093/clipsy.8.3.255

11. Kanter JW, Manos RC, Bowe WM, Baruch DE, Busch AM, Rusch LC. What is behavioral activation? A review of the empirical literature. Clin Psychol Rev 2010;30:608-20.
12. Hopko DR, Bell JL, Armento M, Robertson $\mathrm{S}$, Mullane C, Wolf $\mathrm{N}$, et al. Cognitivebehavior therapy for depressed cancer patients in a medical care setting. Behav Ther 2008;39:126-36. Doi: 10.1016/j. beth.2007.05.007.

13. Hopko DR, Funderburk JS, Shorey RC, Mclndoo CC, Ryba MM, File AA, et al. Behavioral activation and problemsolving therapy for depressed breast cancer patients: preliminary support for decreased suicidal ideation. Behav Modif 2013;37:747-67. Doi: $0.1177 / 0145445513501512$

14. Hopko D, Robertson S, Carvalho JP. Sudden gains in depressed cancer patients treated with behavioral activation therapy. Behav Ther 2009; 40:346-56. Doi: 10.1016/j. beth.2008.09.001

15. Fernández C, Villoria E, Padierna C, Amigo I, Gracia JM, Fernández R, et al. Terapia de activación conductual en pacientes con cáncer. Anal Psicol 2011,27:278-91.

16. Karnofsky DA, Abelman WH, Craver LF, Burchenal JH. The use of the nitrogen mustards in the palliative treatment of carcinoma. Cancer 1948;1:634-56. Doi:10.1002/1097$0142(194811) 1: 4<634$ :: A I D CNCR2820010410>3.0.CO;2-L

17. Sorensen JB, Klee M, Palshof T, Hansen $\mathrm{HH}$. Performance status assessment in cancer patients. An inter-observer variability study. Br J Cancer 1993;67:773-5.

18. Zigmond AS, Snaith RP. The Hospital Anxiety and Depression scale. Acta Psychiatr Scand 1983; 67:361-8. Doi: 10.1111/j.1600-0447.1983.tb09716.x

19. Aaronson NK, Bullinger M, Ahmedzai S. A modular approach to quality of life assessment in cancer clinical trials. Recent Results in Cancer Res 1988;111:231-49.

20. Aaronson NK, Ahmedzai S, Bergman B, Bullinger M, Cull A, Duez NJ, et al. The European Organization for Research and Treatment of Cancer QLQ-C30: A qualityof-life instrument for use in international clinical trials in oncology. J Natl Cancer 
Inst 1993;85:365-76. Doi: 10.1093/ jnci/85.5.365

21. Arrarás JI, Illarramendi JL, Valerdi J. El Cuestionario de Calidad de Vida para cáncer de la EORTC, QLQ-C30. Estudio estadístico de validación con una muestra española. Rev Psicol Salud 1993;7:13-33.

22. Padierna C. Estudio de la calidad de vida de la población oncológica en Asturias [tesis doctoral]. Oviedo: Repositorio Institucional Universidad de Oviedo, Universidad de Oviedo; 2003. Disponible en: http://hdl.handle.net/10651/16570.

23. Livacic-Rojas P, Vallejo G, Fernández P. Power comparison of new tests to analyze repeated measures data. Psicothema 2007;19:673-8.

24. Cabanes A, Pérez-Gómez B, Aragonés N, Pollán M, López-Abente G. La situación del cáncer en España 1975-2006. Madrid: Centro Nacional de Epidemiologia. Instituto de Salud Carlos III; 2009. Disponible en: http://hdl.handle.net/10651/16570.

25. Lejuez CW, Hopko DR, Hopko SD. A brief behavioral activation treatment for depression. Treatment manual. Behav Modif 2001;25:255-86. Doi: 10.1177/0145445510390929.

26. Hopko DR, Armento ME, Robertson SM, Ryba MM, Carvalho JP, Colman LK, et al. Brief behavioral activation and problemsolving therapy for depressed breast cancer patients: randomized trial. J Consult Clin Psychol 2011;79:834-49. Doi: 10.1037/ a0025450.

27. Kaptein AA, Yamaoka K, Snoei L, Kobayashi K, Uchida Y, van der Kloot WA, et al. Illness perceptions and quality of life in Japanese and Dutch patients with non-small-cell lung cancer. Lung Cancer 2011;72:384-90. Doi: 10.1016/j.lungcan, 2010.09.010.

28. Hladschik-Kermer B, Kierner KA, Heck U, Miksovsky A, Reiter B, Zoidl H, et al. Patients and staff perceptions of cancer patients' quality of life. Eur J Oncol Nurs 2013;17:704. Doi: 10.1016/j.ejon.2012.01.005.
29. Padierna C, Fernández C, Amigo I, Gracia J M, Fernández R, Peláez I et al. Estudio longitudinal de los parámetros de calidad de vida en pacientes oncológicos. Psicooncología 2004;1:191-204.

30. Robinson JA, Crawford GB. Do palliative patients and carers agree about patients' psychological functioning? Palliat Support Care 2010;8:69-74. Doi: 10.1017/ S1478951509990721.

31. Villoria E, Fernández C, Amigo I, Padierna C, Peláez I, Fernández R. Calidad de vida en pacientes con cáncer de mama durante el tratamiento oncológico sometidos a intervención psicológica. Evidentia. Index de Enfermería 2011;33:8-11.

32. Borneman T, Koczywas M, Sun V, Piper B, Smith-Idell C, Laroya B, et al. Effectiveness of a clinical intervention to eliminate barriers to pain and fatigue management in oncology. J Palliat Med 2011;14:197205. Doi: 10.1089/jpm.2010.0268.

33. Dhillon HM, van der Ploeg HP, Bell ML, Boyer M, Clarke S, Vardy J. The impact of physical activity on fatigue and quality of life in lung cancer patients: A randomised controlled trial protocol. BMC Cancer 2012;5:572. Doi: 10.1186/1471-2407-12572.

34. Granger $\mathrm{CL}$, McDonald CF, Irving L, Clark RA, Gough K, Murnane A, et al. Low physical activity levels and functional decline in individuals with lung cancer. Lung Cancer 2014;83:292-9. Doi: 10.1016/j.lunG.C.an.2013.11.014.

35. Valdes-Stauber J, Vietz E, Kilian R. The impact of clinical conditions and social factors on the psychological distress of cancer patients: An explorative study at a consultation and liaison service in a rural general hospital. BMC Psychiatry 2013;13:226.

36. Fernández C, Villoria E, Vázquez I, Padierna C, Fernández R, Peláez I. Influencia del estado emocional en la sintomatología referida por pacientes con cáncer de mama y cáncer de pulmón durante el 
tratamiento con quimioterapia. Medicina Paliativa 2013;20:85-92. Doi: 10.1016-j. medipa.2012.05.002

37. Mitchell AJ, Chan M, Bhatti $H$, Halton $M$, Grassi L, Johansen $C$ et al. Prevalence of depression, anxiety, and adjustment disorder in oncological, haematological, and palliative-care settings: A metaanalysis of 94 interview-based studies. Lancet Oncol 2011;12:160-74. Doi: 10.1016/S1470-2045(11)70002-X.

38. Brintzenhofe-Szoc KM, Levin TT, LiY, Kissane DW, Zabora JR. Mixed anxiety/depression symptoms in a large cancer cohort:
Prevalence by cancer type. 2009;50:383-91. Doi: 10.1176/appi.psy.50.4.383.

39. Linden W, Vodermaier A, Mackenzie R, Greig D. Anxiety and depression after cancer diagnosis: Prevalence rates by cancer type, gender, and age. J Affect Disord 2012; 141:343-51. Doi: 10.1016/j. jad. 2012.03.025.

40. Walker J, Holm Hansen C, Martin P, Sawhney A, Thekkumpurath P, Beale C, et al. Prevalence of depression in adults with cancer: A systematic review. Ann Oncol 2013;24:895-900. Doi: 10.1093/annonc/ mds575. 
